\title{
Case studies in post-prostatectomy complications
}

\author{
Greg Bailly, MD, FRCSC \\ Associate Professor and Residency Program Director, Department of Urology, Dalhousie University, Halifax, NS
}

Cite as: Can Urol Assoc J 2013;7(9-10):S195-6. http://dx.doi.org/10.5489/cuaj.1623 Published online October 9, 2013.

\section{Abstract}

As part of the program at the $3^{\text {rd }}$ Annual Canadian Urology Forum (2013), participants engaged in interactive discussions of difficult cases throughout the event. The following is a summary of discussions pertaining to two challenging cases illustrating the management of complications, subsequent to radical prostatectomy.

\section{Case 1}

\section{A 55-year-old man with incontinence post-RP, radiation}

Mr. AC is a 55-year-old carpenter who underwent radical prostatectomy (RP) two years ago, followed by immediate adjuvant radiation.

He subsequently presented with stress urinary incontinence (SUI) with coughing or physical exertion (especially when working). He averaged two to three small pads daily. He had minimal urgency, no urgency incontinence, frequency of three to four micturitions daily and one or two at night. He said that he always had a strong flow when voiding. He was prescribed antimuscarinic therapy (solifenacin), which he indicated was helpful in reducing urgency, but still complained of stress leakage.

\section{Discussion}

The participants discussed how they would work up and manage this patient's "mild" SUI. With respect to nonpharmacologic measures, after some discussion it was thought that the window of opportunity had passed for training and pelvic-floor exercises, as they have only been shown to provide benefit within the first 18 months following RP. These interventions do not influence how continent or incontinent a patient is going to be postoperatively, but rather influence the speed of recovery following the surgery.
Participants did, however, recommend behavioural modification (e.g., timed voiding, regulation of fluid intake) as a potentially useful intervention for this patient.

\section{Case study continued}

In the case of Mr. AC, further testing was ordered to investigate his incontinence. Urodynamic testing showed a maximum cystometric capacity of $500 \mathrm{~mL}$ and bladder compliance was normal, but terminal detrusor activity was noted. There was no obstruction noted, and a post-void residual volume was $60 \mathrm{~mL}$. Cystoscopy was normal, with an open bladder neck.

\section{Discussion}

The participants were asked how the presence of significant storage symptoms would impact their decision-making process with respect to the desirability of surgical intervention. The presence of troublesome storage symptoms in addition to documented SUI should not act as a barrier to going ahead with surgery (e.g., placement of an artificial urinary sphincter [AUS] or male sling). However, patients with this type of profile should be counselled that although the surgery may address the SUI, there may be residual storage symptoms that may require ongoing treatment with an antimuscarinic agent. The decision to recommend an AUS or sling was dependent on the patient's expectations of efficacy, and acceptance of the potential risks of each. Some participants felt that synthetic mesh slings appear to be inferior to the AUS in patients who have had radiation, although data is lacking in the literature.

\section{Case 2}

\section{A 55-year-old man post-RP undergoing adjuvant radiation}

The patient, Mr. MT, experienced bladder neck stricture six weeks after RP. This was treated with dilation using local anesthetic. At four months after RP, he experienced a recurrence of the stricture, which was treated with direct vision internal urethrotomy (VIU) 
and self-catheterization (insertion an 18 French catheter daily). At six months, he began adjuvant radiotherapy.

On the twenty-fifth of his 35 radiation treatments, he was unable to self-catheterize and went into retention. The radiation therapist called the urologist for an urgent consultation.

\section{Discussion}

The participants were asked to discuss the management options for this patient at this point in time. Two options emerged as the participants' preferred choices: 1) to insert a suprapubic catheter and reassess once the radiation is complete, rather than attempt to intervene more aggressively at this point; or 2) insert a Foley catheter and, if tolerated, continue with this option until radiation is complete.

\section{Case study continued}

In Mr. MT's case, he underwent bladder-neck dilation and a Foley catheter was inserted for the duration of his radiation therapy. Following the therapy, he was able to resume self-catheterization initially, but over the next 12 months, he experienced restenosis several times requiring intervention. Management consisted of two more dilations, VIU with eletrocautery, and a transurethral resection of the bladder neck. Following these interventions, Mr. MT was passing bloody necrotic tissue and was completely incontinent. A suprapubic tube was inserted.
At this time, Mr. MT was experiencing severe rectal and pelvic pain, including severe pain on defecation. After consultation with a general surgeon, he was considering a diverting colostomy.

\section{Discussion}

The participants discussed how the presence of rectal and pelvic pain would influence their course of action for a patient with urinary incontinence. It was recommended that a patient like this be seen by pain management specialists, who may be able to recommend measures to reduce his pain beyond analgesia. With respect to the urinary tract, this patient might be best treated with some form of urinary diversion. There was consensus that open reconstruction would not be a viable option in this type of situation, noting that outcomes in a radiated field with this degree of recurrent stenosis is rarely successful. Some participants also felt that the use of a UroLume stent, followed by an AUS, was not advised given the complications seen with UroLume stents under these conditions.

Competing interests: This article is part of a CUAJ supplement sponsored by Astellas Pharma Canada, Inc. Dr. Bailly has received speaker fees, educational grants, and/or travel assistance from Allergan, Astellas, Eli Lilly, and Pfizer.

Correspondence: Dr. Greg Bailly, 620-5991 Spring Garden Rd, Halifax, NS B3H 1Y4; gbailly@dal.ca 